\title{
Editorial
}

Gynäkologe 2014 · 47:321-322

DOI 10.1007/s00129-014-3359-3

Online publiziert: 7. Mai 2014

c) Springer-Verlag Berlin Heidelberg 2014

\author{
R. Kreienberg ${ }^{1}$ H. Hepp ${ }^{2}$ \\ ${ }^{1}$ Landshut \\ ${ }^{2}$ Buch am Ammersee
}

\section{Nachruf zum Tode von Prof. Dr. med. Volker Friedberg}

\author{
05.07.1921-02.03.2014
}

Volker Friedberg, emeritierter ordentlicher Professor für Frauenheilkunde und Geburtshilfe und ehemaliger Direktor der Frauenklinik der Johannes-GutenbergUniversität Mainz, ist am 02.03.2014 im 93. Lebensjahr verstorben. Nach erholsamen Wochen unter südlicher Sonne ereilte ihn der Tod in der Freude auf sein $\mathrm{Zu}$ hause in den Armen seiner Frau Miriam, nachdem ihm als Letztes ein Blick hinab auf die so vertraute und geliebte winterliche Alpenwelt geschenkt war.

Mit ihm hat uns nicht nur eine außergewöhnliche Persönlichkeit verlassen. Mit dem Letzten der fünf Großen der Frauenheilkunde, - Otto Käser, Karl Ober, Klaus Thomsen und Josef Zander - die über Jahrzehnte die Frauenheilkunde und Geburtshilfe gestaltet, geprägt und national wie international zu hohem Ansehen geführt haben, ist eine Ära zu Ende gegangen.

Volker Friedberg wurde am 05.07.1921 als Sohn eines praktischen Arztes in Stuttgart geboren. Nach einer unbeschwerten Schulzeit in Freiburg und Überlingen am Bodensee begann er das Studium der Medizin in Erlangen und Tübingen, legte das Physikum ab und musste danach als Soldat einer Pioniereinheit in den Tiefen Russlands die Schrecken des Zweiten Weltkrieges erleben und erleiden. Noch 1945 konnte er das Studium mit Staatsexamen und Promotion in Berlin abschließen.

Eigentlich zur Inneren Medizin neigend erhielt Volker Friedberg 1945 seine erste (halbe) Assistentenstelle an der
Städtischen Frauenklinik unter dem Direktorat von Prof. Kräuter, dessen Tochter Anneliese, Kollegin aus gemeinsamer Studienzeit in Freiburg, er 1947 heiratete.

Wenn nicht klinischer Internist, so wurde Friedberg ,internistischer Geburtsmediziner“. Seine Habilitation (1954) zum Thema „Über die Ursachen des Schwangerschaftshochdrucks" legte den Grundstein für sein national und international hoch anerkanntes Werk zur Schwangerschaftsgestose.

Die klinische und wissenschaftliche Zeit unter dem Direktorat von Prof. Schwalm nach 1952 empfand Volker Friedberg als herausfordernd und sehr schwierig. Nach dessen Berufung an die Universität Würzburg wurde Friedberg die kommissarische Leitung der Frauenklinik (1959/1960) übertragen. Im Jahr 1960 übernahm Friedberg die Städtische Frauenklinik Saarbrücken, um schließlich nach Berufungen nach Marburg
(1963) und Bonn (1966) am 01. 04.1966 an „seine“ Frauenklinik in Mainz zurückzukehren. Trotz weiterer Berufungen, an die Universitäten Köln, Freiburg und Zürich, blieb Friedberg bis zu seiner Emeritierung 1988 der Johannes-GutenbergUniversität Mainz treu.

Die 22 Jahre seines Direktorats der Universitäts-Frauenklinik Mainz waren geprägt von wissenschaftlicher Neugierde und Offenheit für interdisziplinäre Kooperation. Beispielhaft genannt seien die bereits in den 1960er-Jahren mit dem Pädiater Ewerbeck (Köln) veranstalteten Tagungen in Bad Schachen - Grundstein für die sich entwickelnde Peri- und Neonatologie.

„Pionier“ war Friedberg auch in der gynäkologischen Onkochirurgie. Er hat

Dieser Beitrag erscheint ebenfalls in Ausgabe 6/2014 der Zeitschrift Geburtshilfe und Frauenheilkunde

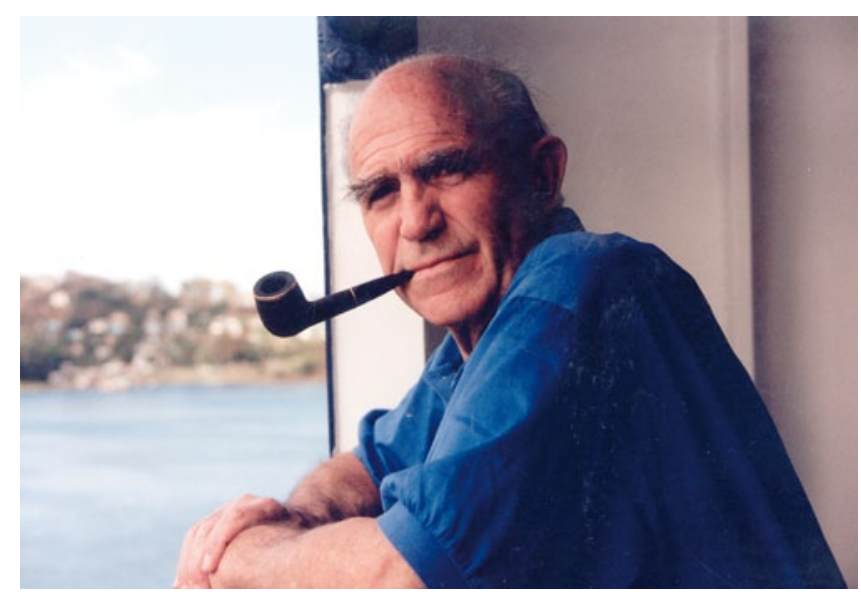

Abbildung privat, mit freundl. Genehmigung 
die operative Senologie einschließlich der plastisch-chirurgischen Mammarekonstruktion "heimgeholt“. Auch in der Onkochirurgie des Beckens hat Friedberg dank seiner operativen Exzellenz und Virtuosität Maßstäbe und Impulse gesetzt. In Indikationsstellung und Operation stets und ausschließlich am Wohl der ihm anvertrauten Patientin orientiert, war er für seine Schüler täglich Lehrer im hippokratischen Sinne. Es gelang ihm durch seine Persönlichkeit, sein Vorbild, seinen Arbeitsstil mit wöchentlichen Visiten und Untersuchung aller zur Operation vorgesehenen Patientinnen, seine ärztlich-ethischen Prinzipien und nicht zuletzt seinen Führungsstil eine „Schu$l^{\prime \prime}$ " zu formen, aus der 7 Lehrstuhlinhaber und zahlreiche Chefärzte hervorgingen, die sein Werk weitergeführt und verbreitet haben.

Führung war für Volker Friedberg Arbeit im Team, gepaart mit - manchmal zu großem - Vertrauen, was Enttäuschungen einschloss. In keiner Situation war sein Führungsstil autoritär, sondern stets von hoher Kompetenz bestimmt. Seine geradezu sprichwörtliche Bescheidenheit verführte ihn gelegentlich, nicht nur bei einer klinisch-wissenschaftlichen Nachfrage, zu dem einleitenden Hinweis, er verstünde von dem zur Diskussion stehenden Sachverhalt wenig, um am Ende des Dialoges den Gesprächspartner erstaunt, oft auch sprachlos ob seiner so fundierten Antwort zurückzulassen.

Eingangs war von einer beendeten Ära die Rede. Diese war auch und vor allem geprägt von der Herausgabe des 3-bändigen Lehr- und Handbuchs "Gynäkologie und Geburtshilfe" im Thieme-Verlag im Jahr 1967 und deren 2. erweiterter Auflage in 1981. Zum Meilenstein einer Fort - und Weiterbildung auf hohem Niveau wurde die von Friedberg mit O. Käser und E.J. Plotz begründete Zeitschrift „Der Gynäkologe“. Viel Kraft, Zeit und Inspiration hat Friedberg ,seinem Kind“ geschenkt.
Volker Friedberg war eine große und profilierte Persönlichkeit. Er hat hohen Anteil daran, dass das Fach Frauenheilkunde und Geburtshilfe in den Nachkriegsjahrzehnten national und international wieder hohes Ansehen erlangte. Dem entsprachen die zahlreichen Ehrungen in Form von Ehrenmitgliedschaften in vielen in- und ausländischen wissenschaftlichen Gesellschaften. As 51-Jähriger wurde er Präsident der Deutschen Gesellschaft für Gynäkologie und Geburtshilfe (DGGG; 1972-1974), später deren Ehrenmitglied. Ferner war er Träger der Carl-Kaufmann-Medaille, der höchsten Auszeichnung der DGGG.

Bewundernswert geglückt ist Volker Friedberg schließlich der Umstieg in das Leben nach dem Beruf. Er vollzog diesen Schritt in der ihm eigenen Konsequenz: Nach 42 Jahren kehrte er mit seiner Frau Miriam aus Mainz in seine allemannische Heimat nach Überlingen/Hödingen zurück, wurde vom Lehrer der Medizin zum Studenten der Philosophie bei Professor Jürgen Mittelstraß an der Universität Konstanz und schrieb eine beachtliche Seminararbeit über das Werk des aus dem Nachbarort Messkirch stammenden Philosophen Martin Heidegger. In schon erwähnter Untertreibung nannte er dieses Studium „vor sich hin heideggern“ und freute sich an Musik, Kunst, Segeln, Bergwandern, Golfspiel und Skilaufen - wann immer möglich auch mit den Söhnen und Enkeln.

Für die zahlreichen Schüler wurde aus dem Lehrer und Vorgesetzten ein kluger Ratgeber, Förderer und väterlicher Freund. Dankbar sind und bleiben wir für die jährlichen Begegnungen und Gespräche im „Engadiner Friedberg-Freundeskreis“.
Nun trauern wir, seine Schüler, seine Freunde des Kollegiums mit seiner Frau Miriam, den Kindern und Enkeln um Volker Friedberg, unseren väterlichen Freund. Wir sagen Miriam vor allem von Herzen Dank für ihre hingebungsvolle, unermüdliche Begleitung und Pflege während der durch körperliche Beeinträchtigung ihres Mannes geprägten vergangenen 4 Jahre.

Wir trauern, gleichzeitig sind wir glücklich und dankbar, dass wir Volker Friedberg begegnen, erleben und ein Stück des Weges gemeinsam mit ihm gehen durften.

Wir verneigen uns in großer Dankbarkeit und tiefer, bleibender Verehrung.

Für die Schüler und Freunde

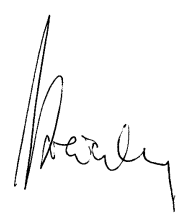

Rolf Kreienberg

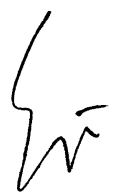

Hermann Hepp

\section{Korrespondenzadressen}

Prof. Dr. R. Kreienberg

Weinzierlstr. 32, 84036 Landshut

r.kreienberg@t-online.de

Prof. Dr. H. Hepp

Schlossstr. 15, 82266 Buch am Ammersee hermann_hepp@gmx.de 